\title{
\begin{tabular}{l|l} 
Mibraries & DSpace@MIT
\end{tabular}
}

\author{
MIT Open Access Articles
}

\section{MIT GEOblog: a platform for digital annotation of space for collective community based digital story telling}

The MIT Faculty has made this article openly available. Please share how this access benefits you. Your story matters.

Citation: Nabian, N., Ratti, C., Biderman, A. and Grise, G. (2009). MIT GEOblog: A platform for digital annotation of space for collective community based digital story telling. 3rd IEEE International Conference on Digital Ecosystems and Technologies, 2009 (Piscataway, N. J.: IEEE): 353-358. (c) 2009 IEEE

As Published: http://dx.doi.org/10.1109/DEST.2009.5276771

Publisher: Institute of Electrical and Electronics Engineers

Persistent URL: http://hdl.handle.net/1721.1/58847

Version: Final published version: final published article, as it appeared in a journal, conference proceedings, or other formally published context

Terms of Use: Article is made available in accordance with the publisher's policy and may be subject to US copyright law. Please refer to the publisher's site for terms of use. 


\title{
MIT GEOblog: A Platform for Digital Annotation of Space for Collec- tive Community Based Digital Story Telling
}

\author{
Nashid Nabian ${ }^{1}$, Carlo Ratti ${ }^{2}$ Member, IEEE, Assaf Biderman ${ }^{3}$, Member, IEEE and Gabriel Grise ${ }^{4}$ \\ ${ }^{1}$ MIT Senseable City Lab, Department of Architecture and Urban Planning, Massachusetts Institute of Technology | Graduate \\ School of Design, Harvard University, Cambridge, MA, USA, e-mail : nnabian@mit.edu \\ ${ }^{2,3,4}$ MIT Senseable City Lab, Department of Architecture and Urban Planning, Massachusetts Institute of Technology, Cam- \\ bridge, MA, USA
}

\begin{abstract}
This paper focuses on guidelines in designing platforms for collective, location-sensitive user generated content, built upon a system that allows for locating mobile subjects within the space. The process of conceptual design, design development, and technical implementation of MIT GEOblog project from a user-interaction design point of view, is used to illustrate the applicability of the guidelines. GEOblog is a web-based platform that allows people to annotate the space, through geo-tagging and sharing user generated content or, in other words, placing digital content over spatial zones that can be retrieved by others based on their real-time sensed location by the system.
\end{abstract}

Index Terms - WIFI locationing, digital annotation, digital story telling, digital enhancement of publicly shared spaces, geo-tagging, locative media

\section{INTRODUCTION}

GEOblog is a web-based platform that allows users to annotate space, through geo-tagging and sharing user generated content. In other words, the user can add digital content over spatial zones, and this content can be retrieved by others based on their real-time location, as sensed by the system. The platform has been implemented online for the MIT campus and is open to MIT community for content contribution and to the general public as a locationsensitive content retrieval/viewing service.

GEOblog was developed at SENSEable City Lab at MIT. It builds on previous research projects at the lab focused on providing location-based services on MIT campus. The MIT campus has over $4000 \mathrm{Wi}-\mathrm{Fi}$ access points (for comparison, city-wide Wi-Fi systems often have hundreds). This allows for the calculation of Wi-Fi-enabled device locations at a high degree of accuracy (several feet) indoors. This fine-grain network is better than GPS locationing systems, which often only work outdoors.

In addition to the added value of overlaying a digital spatial narrative and enhancing spatial perception for individuals who use the platform to contribute their stories or retrieve others, the follow-up analysis of how the system is perceived and received by the community can elucidate the patterns of inhabitation of the physical space and the augmented digital layer's population over time. Also, the study shows how the digital augmentation of space is perceived by its occupants and how it can change the meaning of location, spatial zones and boundary conditions, as well as the concept of temporality in the minds of these individuals.

\section{PROJECT BACKGROUND}

Initially proposed by the MIT Museum, the project was conceptualized as a location-based storytelling research project called Museum With out Walls (MWOW). The original idea was born out of the interest in how locative technologies can link digital information to the physical world. The project proposed a system for a repository of digital information and stories (indexed by location, time and theme) offered by the institute or created by individuals under close supervision of an institutional editorial board. The repository holdings could then be layered over or attached to different spatial content holders around the campus for retrieval by users in proximity, as sensed in realtime by the implemented location-sensing component of the project.

The project initially focused on the "in situ", or "in place" experience of a digital narrative whose various components were attached to different locations on the campus. Here, the individual could retrieve these components while occupying a specific place with a hand-held device that through location sensing technology is aware of its realtime location.

As for location sensing technology, while acknowledging the shortcomings of GPS based location sensing systems that only work outdoors and also experience a drastic decrease in level of their accuracy of determining the realtime location of mobile entities carrying a GPS-enabled device in built areas with high density, the project proposed the use of Wi-Fi base location sensing system for indoor parts of the campus to expand the location-sensitivity of the system to indoors as well as outdoors. The initial idea was to have a Wi-Fi-enabled handheld device to monitor which Wi-Fi access point is being used for wireless internet. Then, based on triangulation of space, where the location of each access node and the signal strength of the available, the system finds nodes and allows the device to calculate its real-time location and retrieve available content for that location.

In the project brief, different system functionalities are illustrated by the graphic below as, "Contribute-StoreExperience." [1].

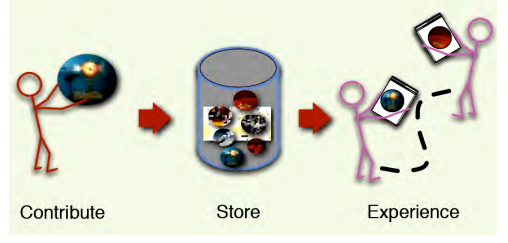


Fig.1 Contribute-Store-Experience, functionalities of the system.

In its initial concept, the proposing party - The MIT Museum - envisioned that the project's content-storing component would be handled on remote content servers. Then, the Wi-Fi-enabled digital device would send a request based on its real-time location to retrieve relevant content.

Also, the goal was to develop the location-sensing service as a device-independent service that could run both on hand-held devices and laptop computers. For content retrieval, the project brief proposed the functionality of an"en situ" content retrieval based on real-time sensed location and distant content retrieval based on users interaction with an on-line map of the campus.

After this proposal was made, the MIT SenseAble City Lab was recruited for further design development .

The Nokia N800 was the proposed handheld device for initial implementation of the prototype. It can connect to internet through a Wi-Fi connection to retrieve content from distant servers, and subsequently calculate a real-time location based on the signal strength of all available Wi-Fi access nodes. This functionality is possible because the geographic location of access nodes are stored on a local file on the device. It also has a touch screen that enhances the user-experience with easy-to-initiate screen-based user input.

MIT SenseAble City Lab was approached by MIT Museum at a time that a platform for location sensing over WiFi network was already implemented and in full operation. The developed platform, iFIND, was unique compared to similar applications, in part because of the extreme precision of its positioning system. More significantly, iFIND was developed with particular attention to privacy and data storage issues. There was no centralized storage of data, and transfers happened through encrypted peer-to-peer transmissions among users. Since the system was devicecentric, as oppose to network-centric, nobody could track another's position unless he enabled this feature [2].

After the initial concept failed to survive for financial reasons, the project transformed into an open platform for digital augmentation of space based on contributions from the MIT community. We called it GEOblog, short for GeoLocalized Platform for Blogging.

\section{DESCRIPTION OF SYSTEM COMPONENTS}

To introduce the platform, we divide the system into different aspects of user interaction and provide a separate description of each aspect., We will also explain the concept behind the conceptual design and implementation factors that have been considered for each component.

User interaction can be broken down into two major categories: content contribution and content retrieval. Incorporating geo-localized campus building floor plans is a part of both the content contribution and content retrieval, as annotating space through geo-tagging, attaching geographical information to a piece of digital content, and placing the content over specific spatial zones feeds into the system's ability to describe the space. This space is then described in terms of the geometry of spatial phenomena as well as actual geographical location of its constitutional elements. Location-sensing will be a sub-category under content retrieval as one possible way of retrieving content is based on the real-time sensed location of an individual on the MIT campus. This technology assumes the user is willing to share his or her location with the system, as the criteria to be used in retrieving relevant content attached to the current location. With this description in mind, the structure of system components can be categorized as follows:

\section{A. Description of System Components -Access Rights and Profile Generation}

Currently, the platform only takes digital content contributions from members of the MIT community. The system is an open platform for user-generated content contribution without a preliminary editorial process to determine the appropriateness of the contributed material. Yet, the concern for content liability over the digital extension of a publicly shared space is still in question. A completely open system that cannot track who is responsible for the placement of certain content, or no content censorship from an institutionally-appointed editorial board, can lead to a social chaos. In this scenario there would be no way to hold system abusers accountable for placing inappropriate or offensive material on the digital extension of a publicly shared space like a university campus. As we can see, the digitallyaugmented space hosts a democratic spatial scenario, since the identity of a contributor is transparent both for the fellow participants by his chosen username and the institution through the track-ability of the user's MIT email account (mandatory for profile generation). Under this arrangement, a user is automatically aware that although he is given the opportunity for un-censored self-expression, he is still held responsible for his actions both by members of the community and the institution authorities. We would call such system a 'controlled democracy', where individuals can express themselves as long as their use of the provided freedom does not result in a hostile or uncomfortable environment for others. Additionally, that this freedom does not violate users' rights to enjoy the digitally-augmented, publicly shared institutional space without feeling discouraged, insulted or intellectually harassed by the content placed on the digital extension of the space. In short, the purpose is to enhance the space, not to impose psychological 'graffiti'.

As mentioned, any individual with a valid MIT email account can register to create a unique user profile on GEOblog. The profile that is created upon registration allows the user to upload digital content and place it over specified zones on campus.

\section{B. Description of System Components -Content Contri- bution and Digital Annotation/Description of the Contributed Content}

Profile holders can contribute digital content after logging into the system. The digital contribution can be in form of digital images or videos:

All popular file formats for digital images are supported by the system. But, due to concerns regarding operational efficiency of the system, the uploaded content will be auto- 
automatically converted to a JPEG format by a server-side function. In addition, all digital contributions are automatically scaled down to fit in the maximum accepted frame by the system: $800 * 600$ pixels in dimensions.

The system allows for uploading multiple digital images as part of a single content contribution. All uploaded digital files will be listed under "Media Files". Once a file is located, clicking "Upload" results in the automatic generation of a thumbnail, to be listed in the "Media Files" section. Clicking on "Delete" on any of the listed thumbnails results in the omission of the digital file from the system.

Furthermore, the automatic resizing of the uploaded digital content is an attempt to address a fundamental restriction of digitally stored spatial annotations, which limit the dimensionality of the faculty of perception in the digital terrain. In the physical terrain, the limits of perceived dimensionality are the sensory faculties: dimensions of the phenomena are perceivable as long as they are within the perceivable range of the sensory faculty. For example, how far can the naked eye see? When perception is limited to the extremities of the digital screen, this given frame becomes the limits of instantaneous perception of phenomena. One approach would be to maintain the original size of the digital image and allow the user to navigate the content using scroll bars, in which case, the image would not be perceived instantly as a whole but through time and as a multiplicity of fragments, each of which is limited to the dimensions allowed by the size of the digital screen. At the conceptual design phase, it was decided that the medium should maintain the integrity of the message - in this case, the digital image- and should offer an overarching view of the phenomena instantly. This then exacts the resizing of the digital images that happen to be bigger than the conventional size of the digital screen, in order to fit in this frame.

In addition to digital images, the user is allowed to contribute video files. At the moment, the only supported format is Flash animation. As soon as a video is uploaded to the system, a thumbnail is automatically generated and listed under "Media Files." Double clicking on the thumbnail will open a conventional video preview window that allows the user to navigate the video.

A possible extension of the system could be incorporating a server-side function to convert any given video format to Flash animation, which allows the system to support other file formats as well. YouTube, the famous and widely used video sharing platform has implemented this functionality and allows users to upload video files of various formats and converts these formats to flash animations before being stored on the system.

On GEOblog, users are offered the opportunity to specify certain YouTube entries as digital content. You Tube, can be accessed by the system to retrieve videos that are already uploaded to this platform.

Once single or multiple digital files are uploaded to the system, this collection is treated as a single entry for which the user is allowed to specify a name, the actual date to which the story corresponds, a temporal span through which the narrative is going to be available for viewing, a multi-line description, and tags separated by commas. Also, the user is allowed to specify who should be granted access to this specific digital narrative. (for example, the MIT community or the general public)

Allowing the user to specify access rights for different digital narratives can be translated as a poetic aspect of the digitally-annotated space that addresses one of the dualities inherent in architectural discourse: the question of domestic/private spaces versus publicly shared spaces. A domestic or private space is differentiated from a publicly shared space based on three major criteria: ownership, access and transparency. Whereas any given domestic space is conventionally privately owned, and is selective to whom access is granted inside both physically as in right of entrance and visually through transparency, a public space is conceptualized as an spatial entity, publicly owned or administered. It is open to the public both in terms of rights of entrance and transparency. Of course, variations in levels of access and transparency results in conceptualization of different categories such as private, semi-private, semi-public or public. As a result, specifying different access rights for different digital narratives is what actually defines how private or public is the digital narrative, and for that matter the digitally annotated space.

If a physical zone is annotated with a multiplicity of digital narratives, each digital narrative redefines the physical space as public or private. The same physical arrangement can be considered public-granting the least level of sensational intimacy if viewed as the holder of a digital narrative accessible to general public, whereas it can also be considered, domestic or private, offering the highest level of sense of intimacy if recognized as the holder of a digital narrative which is not accessible to the public and which is selective about to whom it is revealed.

One possible extension of the system functionality is to add more variations to the access rights which would enable the contributor to be more specific about granting access to the contributed content. Imagine a case where the user can choose if the digital narrative is accessible to friends, close friends, colleagues, classmates in a specific course, or even a list of explicitly specified individuals.

Whereas access rights can introduce parallel variations in terms of privacy and public-ness of a digitally augmented space, specifying a temporal framework through which a digital narrative is portrayed expands the dimensionality of the augmented space along a fourth dimension : time. As opposed to the conventional architectural space that is inherently perceived as fixed and inert, a digitally-augmented space mutates through time. This transformation is perceived by the occupant of such space when he recognizes that a given digital narrative retrieved at a previous incident is no longer there. The digitally augmented layer is constantly transforming through time. The narratives appear and disappear. In terms of user experience, the more one immerses himself in the digital layer, the more he recognizes that this spatial entity has a life of its own. The inherent immutability of the physical context is what gives the subject a sense of spatial orientation. In an evertransforming augmented space, such sense of spatial orientation is not as easily attainable; the spatial context as a per- 
ceptual point of reference is not fixed through time. This subtle spatial disorientation can be considered poetic.

C. Digital Annotation of Space - Attaching Digital Content to Physical Locale

This functionality offered by the platform, allows the user to place digital content within actual physical locations in form of spatial zones. GEOBlog's contribution is a twofold: specifying the spatial zone, and attaching textual information and media files to the created spatial zone. After reviewing online case studies, an interaction scenario was devised for the system that would support the following aspects :

For content contribution, the user should be allowed to upload multiple digital files with one command. It is common sense to give the contributor the capability to devise sets of digital content that represent different aspects or parts of a narrative about a location.

The platform should allow the user to attach digital content or sets of multiple digital contents to zones in space as opposed to geographical nodes. In conventional usergenerated content-sharing platforms, the retrieval of the content happens via a navigable map interface. Thus, the user's spatial perception of the geo-tagged or annotated space is holistic and planar. In this setting, the individual perceives himself as an outsider to the space represented by the navigable annotated map. As a result, it is acceptable to assume that narratives offered by digital content can be attached to discreet nodes on the representational map.

On the contrary, in the GEOblog platform, the goal is to allow participants to retrieve content while corporeally present within the annotated space as measured by their real-time location. In such circumstances, the perception of space is a direct and through sensory experience of the spatial phenomena and not mediated through a map. The subject perceives himself within the annotated space, whereas the subject's perception is based on perspectival views. Spatial entities are received as extensions along physicallydiscernable dimensions. If the space is received as perceivable extensions, the medium that allows for annotation of this phenomena should accommodate the dimensionality of the phenomena as well. As a result, in annotation of a geotaggable space when the retrieval of the geo-tagged content happens through corporeal navigation of the augmented space, the interface should allow the user to put digital content over extensions or spatial zones as opposed to limiting him to attaching this digital content to stand alone nodes. This way the physical boundaries of the spatial phenomena correspond to the virtual boundaries of the digital content which serves as a narrative associated with the space.

In node-based annotation of space using an interactive map like the Google Map API, the space is assumed to be equal to the surface of the globe. On the contrary, as a spherical skin, GEOblog should incorporate the third dimension. GEOblog conceptualizes the space as a 2.5 dimensional entity consisting of multiple floor plans stacked on top of each other.

The user can horizontally navigate through the space by using the zoom and pan functions of the interactive map. Meanwhile, it is possible for the user to navigate across different floors using the virtual elevator tool.
If the space is treated as a 2 dimensional phenomena, no location is an unacceptable for defining a spatial zone as a placeholder for the digital content. On the contrary, once the $3^{\text {rd }}$ dimension is incorporated in the description of the geo-taggable or annotatable space, the created spatial zones should be examined by the system to assure they are accepted within the 3 dimensional space. For example, if a spatial zone is created on the third floor of the building, the system should be able to compare the zone against the extremities of the building's envelope to make sure that the specified zone does not fall outside of this boundary into the void on the third floor. Thus, the system should be able to readjust the spatial zone to acceptable areas. While the offered "zone" tool allows the user to specify a circular or polygonal zone, the system will modify the specified zone in reference to geo-localized plans of MIT buildings and on which floor the user has specified to place the zone. On upper floors, the areas of the created zone outside of the building are automatically omitted.

All aspects of the user interaction should be presented in one coherent view for the user to support a seamless flow of interaction scenario. Content upload and geo-tagging, adding and viewing previously-added semantic information, and placing the content on the map, are all incorporated in one convenient view.

As a result of implementing the aformentioned design criteria, digital content contribution occurs in 5 steps, in the bounds of one comprehensive screen that accommodates all steps at once.

[1] Specifying the spatial zone over which content is placed with the interactive navigatable map interface; [2] Uploading the digital file containing the shared content. (Content in form of digital image and video are supported by the platform); [3] Specifying the duration through which the content will be available for retrieval; [4] Adding semantic information or metadata about the shared content including title, description and semantic tags; [5] Specifying access rights and whether the geo-tagged/shared content should be available to everybody on campus, just the members of MIT community or the owner of the content.

After an entry is completed by the user it would be added to the list of previously completed entries. Once the augmented digital layer is populated by contributed content, participating parties can retrieve them in three different ways: by semantic search; interactive navigable map interface; and on self-initiated, real-time user location reporting.

By default, the participant's real-time location is calculated based on IP address, which indicates the campus building the participant occupies. As a result, the system would locate the participant at the middle of that building. Using a java applet in combination with "Find Me" functionality provides a precise location, because it continuously screens and reports the Wi-Fi Access Points' Mac Address, and signal strength from which the participant is connected to wireless network.

\section{GEO-BLOG TECHNICAL IMPLEMENTATION - GEOGRAPHIC DESCRIPTION OF SPACE}

GEOblog is a location-based content contribution and 
retrieval system. By definition, such system needs to be implemented using GIS technology. In GEOblog, multiple GIS tables are incorporated in the architecture of the system:

The first table is populated by geo-localized floor plans of all the buildings in MIT Campus. Each location is a recognizable boundary, or footprint, and is represented as a unique entry in this data set. Each record in the dataset includes the geometry of the location in form of a twodimensional polygon and the name of the space according to the naming convention that is consistent all over the campus - i.e. w85-1201 refers to the room 201 on the first floor in building w85; and the type of the space (whether it is a classroom, a corridor or connecting space, a service area, a student residence, a lecture hall or a lobby).

The second table is populated by the location of all the Wi-Fi access points around the campus. Each entry in this dataset includes the following information about the access node it represents: The unique mac address of the node, the name of the node, the name of the building in which the node is installed, the floor or building level on which the node is installed, the name of the room or space at which the node is located, and the geographical location of the node in form of a point object.

Once a zone is created by a participant as the place holder of a digital file, an entry is created in a third dataset. This entry includes the following information about the spatial zone that it represents: a unique identification number that helps the system to refer to the entry, a binary field that specifies if the zone is a circular one- if not, the system will assume that it is a polygonal feature, a reference field that associates the spatial zone to the digital content that is being attached to it and is stored in another data table - the data table that all the digital files are stored in - and finally the actual geometry of the created zone in form of a polygonal object. When the user creates the zone, the system compares the boundaries of the zone with the geo-localized floor plan and subtracts areas that fall outside the building envelope.

With the first dataset, any given point on campus is geographically described, and via the third dataset, any spatial zone that is created by the user is interpreted by the system as a geographical entity. Next, the digital content that is attached to this defined spatial zone by the user is automatically associated with geographical locations on the campus.

On the other hand, for the content retrieval aspect of the project, if the user chooses to retrieve content based on selfreported real-time location, a java applet will monitor all available Wi-Fi access nodes and send a list of the mac addresses of the available access nodes, as well as the wireless signal strength of each of them to the server. The system calculates the real-time location of the self-reporting entity using a spatial triangulation procedure based on the provided data. The following graph shows the principals of location sensing based on spatial triangulation:

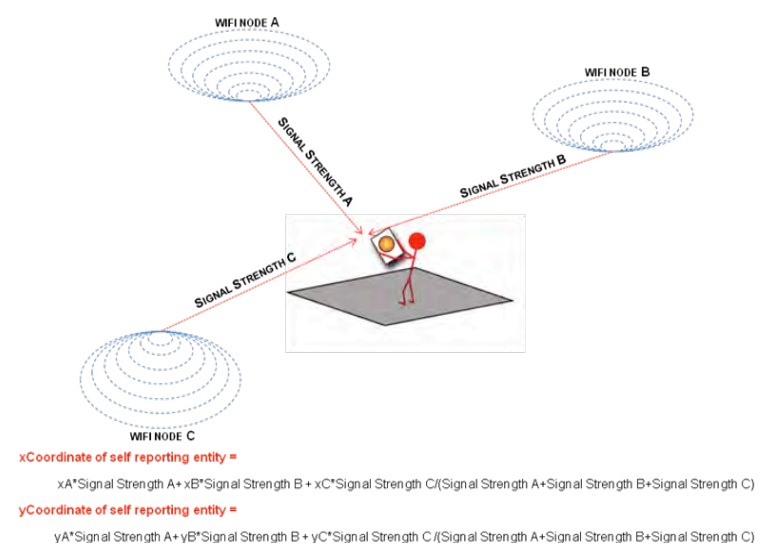

Fig.2 Spatial triangulation based on Wi-Fi network and self-reported information of the Wi-Fi enabled device.

When the location of the self-reporting agent is calculated as a geographical point with known longitude and latitude, the location of the point can be compared against the geographical description of the spatial zones stored that contain digital contributions. The system will then automatically identify the spatial zones that contain the calculated geographical point that represents the real-time location of the self-reporting agent. Consequently, the digital content that is associated with these spatial zones will be retrieved from the database and made available for viewing.

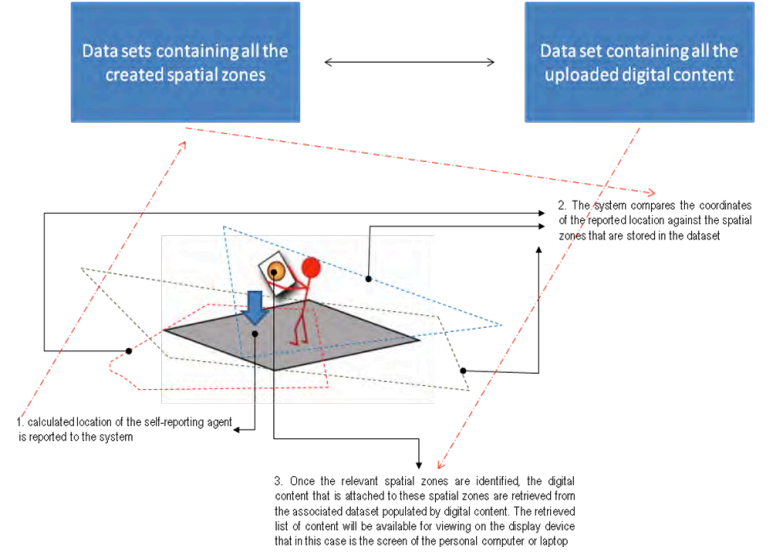

Fig.3 Graph illustrating how content is retrieved based on locational criteria

The following graph illustrates how the system uses each table and how records are associated across different tables:

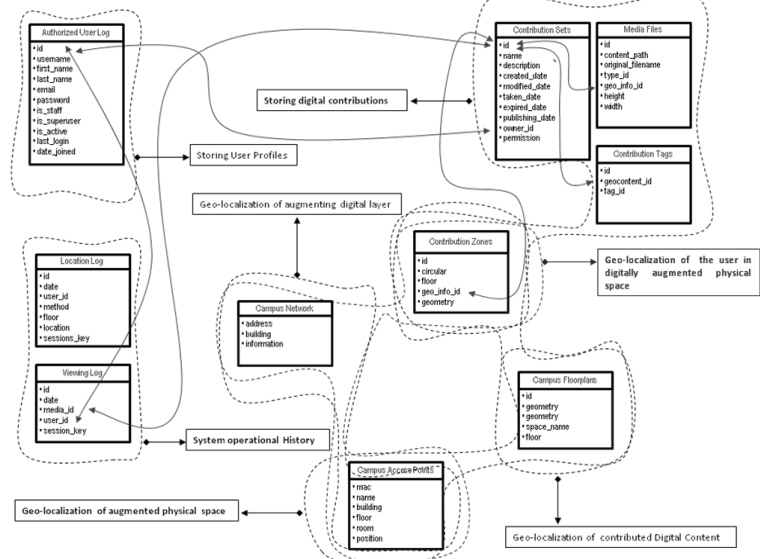

Fig.3 GEOblog database scheme 


\section{GEOBLOG - FutURE DiRECTIONS}

In this last section we would like to focus on future directions and possibilities of the implemented system. The implemented system, although complete and operational, is open to future expansion and improvement, under the following context-sensitive adaptations:

1. Expandability of the system via new additions to GeoServer entries and other datasets, and applicability of the system to different [institutional] sites:

GEOblog uses three different geo-spatial tables to geolocalize the physical space that is used as a frame of reference while contributing and geo-tagging content. Meanwhile, the user self-reports his/her real-time location to retrieve relevant content in situ:

i. A "Campus Network Data Table" that offers a list of all campus wireless sub-networks and the range of dynamic IP addresses that are assigned to a system that connects to a network. This network is unique for each MIT Building, which allows for a low- accuracy locationing based on reverse geo-coding of IP address of the user that is connecting to the GEOblog server.

ii. A "Campus Access Points Data Table" that in collaboration with an applet allows the user to self-report his real-time location with a high resolution based on Wi-Fi wireless network of the campus. This table consists of all Wi-Fi access nodes and their geographical longitude and latitude.

iii. A "Campus Floor Plan Data Table" which is a table populated by all the geo-localized floor plans of the buildings in the campus. The entries of this data table will populate a navigatable/interactive map interface as a reference frame for adding digital content over specified spatial zones and specifying physical location on the map to retrieve relevant content placed over/attached to the specified locality.

It is obvious that all these three data tables are sitespecific and their content will differ depending on the site. However, the system has been designed to be independent from the tables' content. This means that for a new site, if these three site-specific datasets are updated with pertinent information, the system will automatically adapt to the specificities of the new site. Based on the same reasoning, after the GEOblog platform is employed for a given site, it will function to its fullest capabilities. In case the project site expands, for example, due to further real-state acquisition, the digital landscape of the site changes, Wi-Fi access nodes are omitted, or there is change in how dynamic IP addresses are assigned, the datasets can be updated along with the modified or extended digitally-augmented landscape.

2. Improvement of Location-Sensing Technology through incorporation of the material properties of architectural elements, and/or incorporation of tracking the self reporting agent in the space through time:
The wireless signal strength drastically changes in its attributes while travelling through different architectural elements with different material properties. These properties can affect the precision of location-sensing procedure. Incorporation of material characteristics of the architectural elements as a field entry in the Geo-Localized Floor Plans dataset, and using this data in the location-sensing procedure will result in achieving maximum level of precision.

Furthermore, it is also possible to improve the precision of the system if each self-reported or sensed location is compared by the system to the trajectory of the movement of the mobile agent through space. A drastic change in location, sensed over a short time interval, can be the result of a possible error in location-sensing. In this event, the system can be designed to correct itself. The system can learn from such incidents to develop sensitivity to the areas that frequently report these errors or inaccuracies.

3. Further Enhancement of User Experience, Benefiting from the Implemented technological platform: With minor changes or additions to the implemented platform, other scenarios that enhance the user experience in a digitally augmented physical space can also be supported.

i. Virtual Hopscotch: This interaction mode allows the participant to navigate the data space, as overlaid on a physical space. This virtual experience is different from the immediate space that he/she inhabits, based on the roaming of a fellow participant in another location. Any participant owns a virtual avatar and a virtual shadow of her/his own. The virtual avatar represents the normalized location of the participants in the augmented space and the shadow represents the normalized location of the participant in the datascape. By dragging one's shadow to the location of another participant's avatar, one can initiate a request to virtually hopscotch to the other subject's location in the data-scape, and gain access to his resources, as allocated by his existence in his space.

ii. Digital Story Lines: Right now, the platform allows for contributing stand-alone instances of an accordance in form of digital media that has documented it. If a tracking system is also implemented, the users can also contribute story lines that consist of multiplicity of story zones along a defined path. When retrieving content, the user can specify that $\mathrm{s} /$ he wants to retrieve digital content that pertain to a specific story along a path. The system can then guide the user through the space to corporeally follow the story that consists of milestones along a path in the physical space.

\section{REFERENCES}

[1] http://museum.mit.edu/mwow

[2] Huang, S., Proulx, F. \& Ratti, C. (2007) "iFIND: A Peer-to-Peer Application for Real-Time Location Monitoring on the MIT Campus," Proceedings of the CUPUM 07 - 10th International Conference on Computers in Urban Planning and Urban Management, Iguassu Falls, 11-13 July, 2007. 